\title{
The moderating role of COMT Val158Met polymorphism in the relation between maternal rearing style and loneliness: implications for mental health
}

\author{
Wen Wei, Yudong Lin, Tiantian Hong, and Siyang Luo \\ Sun Yet-san University \\ Department of Psychology
}

\begin{abstract}
Loneliness is a common problem in adulthood, with deleterious effects on mental health conditions. In the present study, we aimed to examine the predictors of loneliness, with a focus on genetic factors (COMT genotype) and maternal rearing style, and further consider how loneliness moderates the relationship between maternal rearing style and adult's mental health. The present results showed that COMT genotype significantly moderated the relationship between maternal rejection and loneliness, while only individuals who perceived reject from their mothers and carried $\mathrm{Val} / \mathrm{Val}$ genotype were at increased risk for developing loneliness. When considering the moderated mediation model, current findings demonstrated that COMT genotype moderated the indirect effects of maternal reject and social anxiety as well as life satisfaction, and these effects are significant cross-age. Specifically, with Val/Val genotype, individuals who received a high rejection from the mother would influence their mental health through loneliness. These findings contribute to a further understanding of the environmental and genetic basis of loneliness and its influence on adulthood.
\end{abstract}

Keywords loneliness, COMT Val158Met, maternal rearing style, gene-environment interaction, mental health.

\section{Introduction}

Loneliness is typically defined as the subjective feeling of dissatisfaction that occurs when one's actual social relationships are inadequate in light of one's desired or ideal social relationships (Luo, Hawkley, Waite, \& Cacioppo, 2012). The percentage of people reporting feeling lonely "sometimes" or "often" is estimated to be between $11 \%$ and $30 \%$ of adults (Dykstra, Van Tilburg, \& Gierveld, 2005; Hawthorne, 2008; Griffin, 2010). Evidence has shown that loneliness is related to various adverse health conditions, including increased cardiovascular health risk (Caspi et al., 2006) and depression in adults (Cacioppo, Hawkley, \& Thisted, 2010). Therefore, it is important to examine its antecedents and its outcomes. The present study aimed to examine the predictors of loneliness, with a focus on maternal rearing style and its relation with genetic factors 
(COMT genotype), and further consider how loneliness moderates the relationship between maternal rearing style and adult's mental health.

The evolutionary theory of loneliness (ETL) posits that loneliness is influenced by both environmental factors and genetic inheritance (J.T. Cacioppo et al., 2014; J. T. Cacioppo \& Patrick, 2008). As for environmental factors, a meta-analysis (Mahon et al., 2006) revealed that the most important environmental predictors of loneliness were social support and parental support. Prior research has found that the buffering role of social support varies by its sources in that parental support, not peer support, buffers the adverse association between stress and individuals' psychological well-being (Crockett et al. 2007). In specific, the different effects of maternal versus paternal support demonstrated the necessity of separating individual types of support, rather than combining them as in previous research (Chao, 2012; Lee et al., 2014), and highlighted the importance of maternal rearing style. For example, mothers spend considerably more time socializing children compared to men (Yeung et al., 2001). Also, Xu et al. (2017) found that emotional warmth, overprotection and rejection were significantly more often perceived from mothers than from fathers. So, the current study focuses on maternal rearing style and expects that maternal rearing style is related to loneliness.

Behavioral genetic studies have found a significant genetic component for loneliness, with estimates ranging between $37 \%$ and 55\% (Goossens et al, 2015). Several studies have found a significant relation of a particular gene to loneliness (Lan et al., 2012; Tsai et al., 2012; Lucht et al., 2009). However, loneliness is a complex trait that is affected by numerous forms of genetic influence, indicating the involvement of additional genetic variants in the expression of loneliness. In the context of the dopaminergic neurotransmission, especially the Catechol-O-methyltransferase (COMT) Val158Met polymorphism is an interesting candidate polymorphism because this gene locus has turned out to be functional. The existing research has not examined the direct effects of the COMT gene on loneliness. Loneliness is said to be a significant variable affecting depression (Erzen \& Çikrikci, 2018) and also play a causal role in the development of depression (Yavuzer, Albayrak, \& Kılıçarslan, 2019). Many studies have reported an association of the COMT Val158Met polymorphism and depression, although with conflicting results (Klein, Schmoeger, Kasper, \& Schosser, 2016). Therefore, we expected that the COMT 
Val158Met polymorphism genotype would be related to loneliness and could be regarded as a candidate gene.

The ETL also states that some people are more likely to experience loneliness than others. This individual variability in loneliness is affected by both genes and the environment or, to be more precise, by their interaction. Ira et al. (2014) found that individuals exposed to low maternal care as a child (childhood adversity) and who experienced stressful life events in adulthood (proximal stress) reported more psychotic symptoms, and this relationship was strongest among the Val/Val COMT group. Also, the association between stressful life events and bipolar disorder is significant and higher for those in the Val dominant group compared to those with the Met/Met genotype (Hosang et al., 2017). Drury et al. (2010) also found evidence of a COMT Val158Met $\times$ early severe social deprivation interaction on depressive symptoms. So, we expected the COMT Val158Met to interact with environmental factors in predicting loneliness.

The evolutionary theory not only frames loneliness as an important outcome but also frames loneliness as an important intermediate variable predicting other mental health outcomes. For example, Goodwin et al. (2001) found that loneliness is negatively related to life satisfaction. A diary study has shown that individuals high on loneliness experienced less reward from daily social interactions compared with individuals low on loneliness (Hawkley, Preacher, \& Cacioppo, 2007). As for social interactions, lonely people are identified to have more negative self-evaluations that promote their perception and anticipation of rejection from others (Jones, Freemon, \& Goswick, 1981), thus having more problems interacting with people, such as social anxiety. In a cross-lagged structural equation model controlling for trait levels and prior states, researchers indicated that earlier loneliness positively predicted future states of social anxiety (Lim, Rodebaugh, Zyphur, \& Gleeson, 2016). Thus, we hypothesized that loneliness might further negatively impact the level of life satisfaction and social anxiety.

In the present investigation, we first examined the relationships of the maternal rearing style, COMT genotype, and COMT by maternal rearing style interactions with loneliness. For the gene-environment interactions, we expected the maternal rearing style to interact with the COMT genotype in predicting loneliness. As the adult child grow up and require less direct care, child-care assistance from parents varies the most dramatically across 
adulthood (Cooney, \& Uhlenberg, 1992). So, it was of particular interest to determine whether and how this interaction would change across age. Then, we examined the role of COMT genotype as a moderator and loneliness as a mediator of the relationship between maternal rearing style and mental health-related consequences.

\section{Methods}

\section{Participants}

We used a Chinese DNA dataset of participants who had consented to participate in scientific research, and we finally have 1034 participants, among which 532 were male $(51.5 \%)$ and 502 were female (48.5\%). The mean age of subjects in this dataset was 26.77 years $(\mathrm{SD}=5.19)$. Using $\mathrm{G}^{*}$ Power 3.1 , there is a $80 \%$ chance of correctly rejecting the null hypothesis of no difference with a total of 155 participants (Faul, Erdfelder, Buchner, \& Lang, 2009).

\section{Genotyping}

COMT Val158Met, which is located at the q11 band of human chromosome 22 (rs4680), results in 3- to 4-fold reduction in COMT enzyme activity by coding for the synthesis of the amino acid methionine (Met) instead of valine (Val). Genomic DNA was extracted from participants' saliva. Both genotyping call rates and concordance for duplicate samples were $>0.95$. The genotype distribution of COMT Val158Met $(\mathrm{N}=544$ $\mathrm{Val} / \mathrm{Val}, \mathrm{N}=404 \mathrm{Val} / \mathrm{Met}, \mathrm{N}=86 \mathrm{Met} / \mathrm{Met}$ ) did not deviate from Hardy-Weinberg equilibrium $(P>0.3)$.

\section{Measures}

Loneliness

SELSA-C. A Chinese variant of the Social and Emotional Loneliness Scale for Adults (SELSA-C) was used to assess loneliness among adults, derived from those of DiTommaso and Spinner (1993). The SELSA-C is a self-reporting questionnaire that is composed of 18-tem subscales: the social loneliness subscale and the emotional loneliness subscale. Using a five-point Likert scale ranging from 1 (strongly disagree) to 5 (strongly agree), the 
participants were asked to report the extent to which these statements apply to them. The Cronbach's $\alpha$ coefficient of SELSA-C loneliness scale was 0.89 .

\section{Maternal rearing style}

$S$-EMBU-C. The Chinese version of the simple parenting style scale (S-EMBU-C) revised by Jiang et al. (2010) was selected. The questionnaire included two parts of the father/mother version, each with 21 questions. The father and mother subscales are composed of three dimensions: rejection, emotional warmth and overprotection. The scale adopts Likert 5-point scoring method, from "never" to "always". Its internal consistency coefficients were 0.844 (reject), 0.831 (overprotection) and 0.873 (emotional warmth) respectively. The higher the average score in each dimension of the questionnaire, the more the mothers adopted the parenting style represented by this dimension.

\section{Social Anxiety}

IAS. The 15-item Interaction Anxiousness Scale (IAS; Leary, 1983) measures people's anxiousness in social situations. The scale adopts Likert 5-point scoring method, from "extremely inconsistent" to "extremely consistent", and the scale is scored $1 \sim 5$ points respectively. Its internal consistency coefficient was 0.909 . The higher the score of the questionnaire represented the more social anxiety level.

\section{Life satisfaction}

SWLS. A 5-item Satisfaction with Life Scale (SWLS) developed by Diener et al. (1985) was used to measure respondents' life satisfaction. Using a 7-point scale, respondents were asked their level of agreement ranging from 1 (strongly disagree) to 7 (strongly agree). Its internal consistency coefficient was 0.899 .

\section{Data analyses}

To minimize systematic variance not due to the variables of interest (Koch \& McGarth, 1996) and thereby enhance internal validity in our study, participants' gender, as well as subjective socioeconomic status (SES), were included as the control variables in all steps of our analysis. A single item was used to measure the demographic characteristics of gender and SES. As the number of scale points increases, ordinal data behave more closely to interval data (Boomsma, 1987; Rigdon, 1998). Each maternal rearing behavior (reject, 
emotional warmth, overprotection) was used as a continuous variable in the models. Firstly, Pearson correlations were used to make descriptive statistics for each variable.

A moderated hierarchical regression analysis was conducted to investigate the effects of COMT Val158Met polymorphism and maternal rearing style on participants' loneliness scores. The interaction terms between each dimension of maternal rearing style and COMT Val158Met genotype were calculated by multiplying the normalized variables together (Aiken, West, \& Reno, 1991). In Step 1 of the regression, gender (dummy code gender: male $=0$ and female $=1$ ), age, subjective socioeconomic status and the other two types of maternal rearing style variables were entered simultaneously to control for the influence of each participant's subjective social-economic status and the influence (such as emotional warmth and overprotection). In Step 2, we entered COMT Val158Met genotypes (dummy code COMT. $\mathrm{Val} / \mathrm{Val}=0, \mathrm{Val} / \mathrm{Met}=1, \mathrm{Met} / \mathrm{Met}=2$ ) as well as the type of maternal rearing style (such as reject) as the independent variable. In Step 3, the two-way interaction term (such as maternal reject $\times$ COMT Val158Met, Fisher r-to-z transformation before computed the interaction term) was entered. Besides, to increase our understanding of how loneliness influence participants' interpersonal skill (social anxiety) and well-being (life satisfaction) at different ages, we split the age variable into three groups to repeat the interactive analysis.

Then we built a moderated mediation model (Figure 1). To examine the moderated mediation model, we used the PROCESS 3.2 for SPSS developed by Hayes (2013) to analyze the hypothesized paths among all ages. Model 7 of the PROCESS was employed in the current study. The covariate variables were age, gender, subjective socioeconomic status, emotion warmth and overprotection. This model produces multiple coefficients: the direct effect of maternal reject $(\mathrm{X})$ on social anxiety $\left(\mathrm{Y}_{1}\right)$ or life satisfaction $\left(\mathrm{Y}_{2}\right)$ (c path) controlling for loneliness, the effect from maternal reject $(\mathrm{X})$ to loneliness (Mediator; $\mathrm{a}_{1}$ path), the effect from COMT (Moderator) to loneliness (Mediator; a path), and the conditional effect of COMT (Moderator) on maternal reject $(\mathrm{X})$ to loneliness (Mediator; $\mathrm{a}_{3}$ path). This analysis analyzes conditional indirect effects of maternal reject on social anxiety/ life satisfaction through loneliness. Indirect effects were tested using 5,000 bootstrapped resamples. This procedure produces a 95\% bias-corrected and adjusted confidence intervals (CIs). Significant indirect effects are identified by CIs that do not 
include zero. The predictor and moderator variables were mean-centered for the conditional effect test. Following Hayes (2015), we also calculated the index of moderated mediation to examine differences between conditional indirect effects. When the index is significantly different from zero, this indicates that the conditional indirect effects are different from each other.

The conditional indirect effect of maternal reject to life satisfaction (social anxiety) through loneliness for indirect effects at three levels of COMT (Val/Val, Val/Met and Met/Met) was conducted. Finally, we repeated the splitting age group analysis. The current study utilized the statistical software SPSS 23.0 and its PROCESS for analysis.

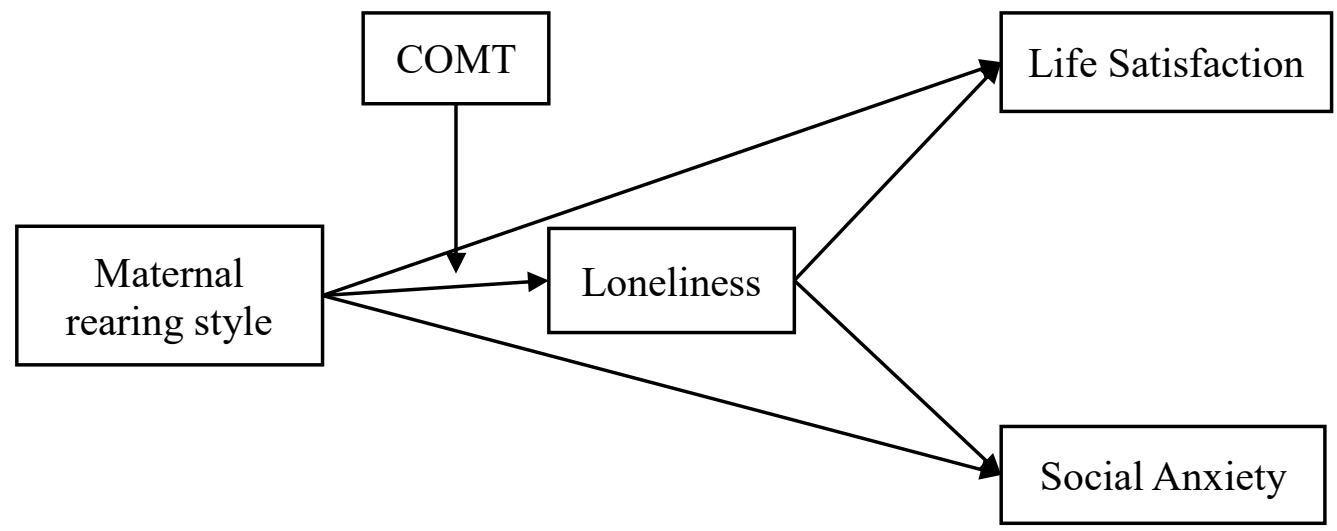

Fig. 1 The proposed moderated mediation model.

\section{Results}

The demographic information, loneliness scores and social anxiety scores of three genotype groups were shown in Table 1. Correlations among variables were presented in Table 2. One-way ANOVAs were conducted to examine the difference in the demographic information among the three variants of COMT Val158Met and the results showed that the allelic distribution was comparable across all variables.

Table 1. Demographic information (mean $\pm \mathrm{SD}$ ) of three genotype groups.

\begin{tabular}{|c|c|c|c|c|c|c|c|c|c|c|c|c|c|c|}
\hline & \multicolumn{3}{|c|}{ Total } & \multicolumn{3}{|c|}{ Val/Val } & \multicolumn{3}{|c|}{ Val/Met } & \multicolumn{3}{|c|}{ Met/Met } & \multirow{2}{*}{$\frac{F}{0.481}$} & \multirow{2}{*}{$\frac{p}{0.618}$} \\
\hline Gender & 531 & 1,50 & & 284 & $\overline{\mathrm{M}, 2}$ & $0 \mathrm{~F}$ & 208 & $\overline{M, 13}$ & & & $\overline{4,46}$ & & & \\
\hline Age & 26.77 & \pm & 5.19 & 26.69 & \pm & 5.13 & 26.79 & \pm & 5.16 & 27.16 & \pm & 5.75 & 0.305 & 0.739 \\
\hline SES & 4.98 & \pm & 1.49 & 4.98 & \pm & 1.47 & 4.93 & \pm & 1.56 & 5.29 & \pm & 1.29 & 2.023 & 0.130 \\
\hline Reject & 9.20 & \pm & 3.20 & 9.39 & \pm & 3.30 & 8.94 & \pm & 3.06 & 9.25 & \pm & 3.18 & 2.298 & 0.107 \\
\hline Emotional Warmth & 16.38 & \pm & 4.80 & 16.47 & \pm & 4.93 & 16.40 & \pm & 4.69 & 15.78 & \pm & 4.54 & 0.768 & 0.463 \\
\hline Loneliness & 46.36 & \pm & 10.04 & 46.51 & \pm & 10.37 & 45.95 & \pm & 9.90 & 47.38 & \pm & 8.43 & 0.840 & 0.429 \\
\hline Social Anxiety & 50.17 & \pm & 10.58 & 50.15 & \pm & 10.60 & 50.24 & \pm & 10.33 & 49.95 & \pm & 11.72 & 0.024 & 0.976 \\
\hline
\end{tabular}


Life Satisfaction

$16.78 \pm 6.73$

$16.82 \pm 6.83$

$16.73 \pm 6.66$

$16.82 \pm 6.47$

0.020

0.980

SES, Subjective Economic Status.

Table 2. Correlations among variables

\begin{tabular}{|c|c|c|c|c|c|c|c|c|c|c|}
\hline Variable & 1 & 2 & 3 & 4 & 5 & 6 & 7 & 8 & 9 & 10 \\
\hline 1. Gender $^{\mathrm{a}}$ & 1 & & & & & & & & & \\
\hline 2. Age & -0.019 & 1 & & & & & & & & \\
\hline 3. SES & 0.056 & $.104^{* *}$ & 1 & & & & & & & \\
\hline 4. Reject & $.131^{* *}$ & -0.008 & $-.097^{* *}$ & 1 & & & & & & \\
\hline 5. Emotional Warmth & $.140^{* *}$ & $-.063^{*}$ & $.226^{* *}$ & $-.140^{* *}$ & 1 & & & & & \\
\hline 6. Overprotection & -0.015 & -0.054 & -0.052 & $.513^{* *}$ & -0.060 & 1 & & & & \\
\hline 7. COMT Val158Met ${ }^{\mathrm{b}}$ & 0.025 & 0.022 & 0.031 & -0.045 & -0.031 & -0.026 & 1 & & & \\
\hline 8. Loneliness & -0.058 & -0.035 & $-.270^{* *}$ & $.249^{* *}$ & $-.300^{* *}$ & $.171^{* *}$ & 0.001 & 1 & & \\
\hline 9. Social Anxiety & 0.027 & $-.074^{*}$ & $-.183^{* *}$ & $.088^{* *}$ & $-.190^{* *}$ & $.148^{* *}$ & -0.001 & $.372^{* *}$ & 1 & \\
\hline 10. Life Satisfaction & $.106^{* *}$ & 0.009 & $.428^{* *}$ & $-.136^{* *}$ & $.303^{* *}$ & $-.105^{* *}$ & -0.003 & $-.448^{* *}$ & $-.241^{* *}$ & 1 \\
\hline
\end{tabular}

$N=1034$.

${ }^{\mathrm{a}} 0=$ boy; 1 = girl.

${ }^{\mathrm{b}} 0=\mathrm{GG}(\mathrm{Val} / \mathrm{Val}) ; 1=\mathrm{AG}(\mathrm{Met} / \mathrm{Val}) ; 2=\mathrm{AA}(\mathrm{Met} / \mathrm{Met})$.

${ }^{*} p<.05 ;{ }^{* *} p<.01$.

COMT, Catechol- $O$-methyltransferase; SES, Subjective Economic Status.

COMT moderated relations between Maternal rearing style and Loneliness

The hierarchical regression analyses first confirmed that the interaction of COMT Val158Vet and maternal reject score predicted participants' loneliness scores (Table 3). Separate analyses revealed significant correlations between loneliness scores and maternal reject in two genotype groups (Val/Val: $\beta=0.315, R^{2}=0.099, P<0.001$; Val/Met: $\beta=$ 0.176, $\left.R^{2}=0.031, P<0.001\right)$. Correlations were not significant in Met/Met carriers (Met/Met: $\beta=0.088, R^{2}=0.008, P=0.423$; Figure $2 \mathrm{~A}$ ). There was a significant correlation between loneliness scores and maternal emotional warmth in the three genotype groups (Val/Val: $\beta=-0.29, R^{2}=0.099, P<0.001$; Val/Met: $\beta=-0.323, R^{2}=0.031, P<$ 0.001; Met/Met: $\beta=-0.25, R^{2}=0.031, P<0.001$; Figure $2 \mathrm{~B}$ ), and the hierarchical regression analyses failed to show significant moderation effect (Table 3). The hierarchical regression analyses also failed to reveal that the interaction of COMT Val158Met genotype and maternal overprotection (Table 3). Moreover, the association between maternal overprotection and loneliness were all significant in all genotype groups ( $\mathrm{Val} / \mathrm{Val}: \beta=$ 0.176, $R^{2}=0.099, P<0.001 ; \mathrm{Val} / \mathrm{Met}: \beta=-0.323, R^{2}=0.031, P<0.001$; Met $/ \mathrm{Met}: \beta=$ $0.140, R^{2}=0.031, P<0.001$; Figure 2 C). 
Maternal support $\times$ COMT
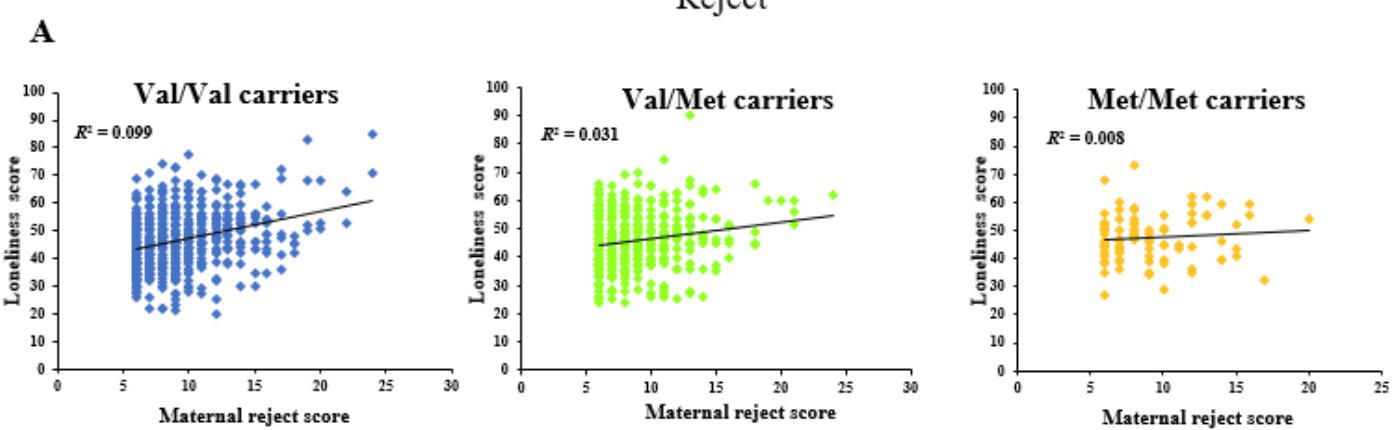

$\mathbf{B}$
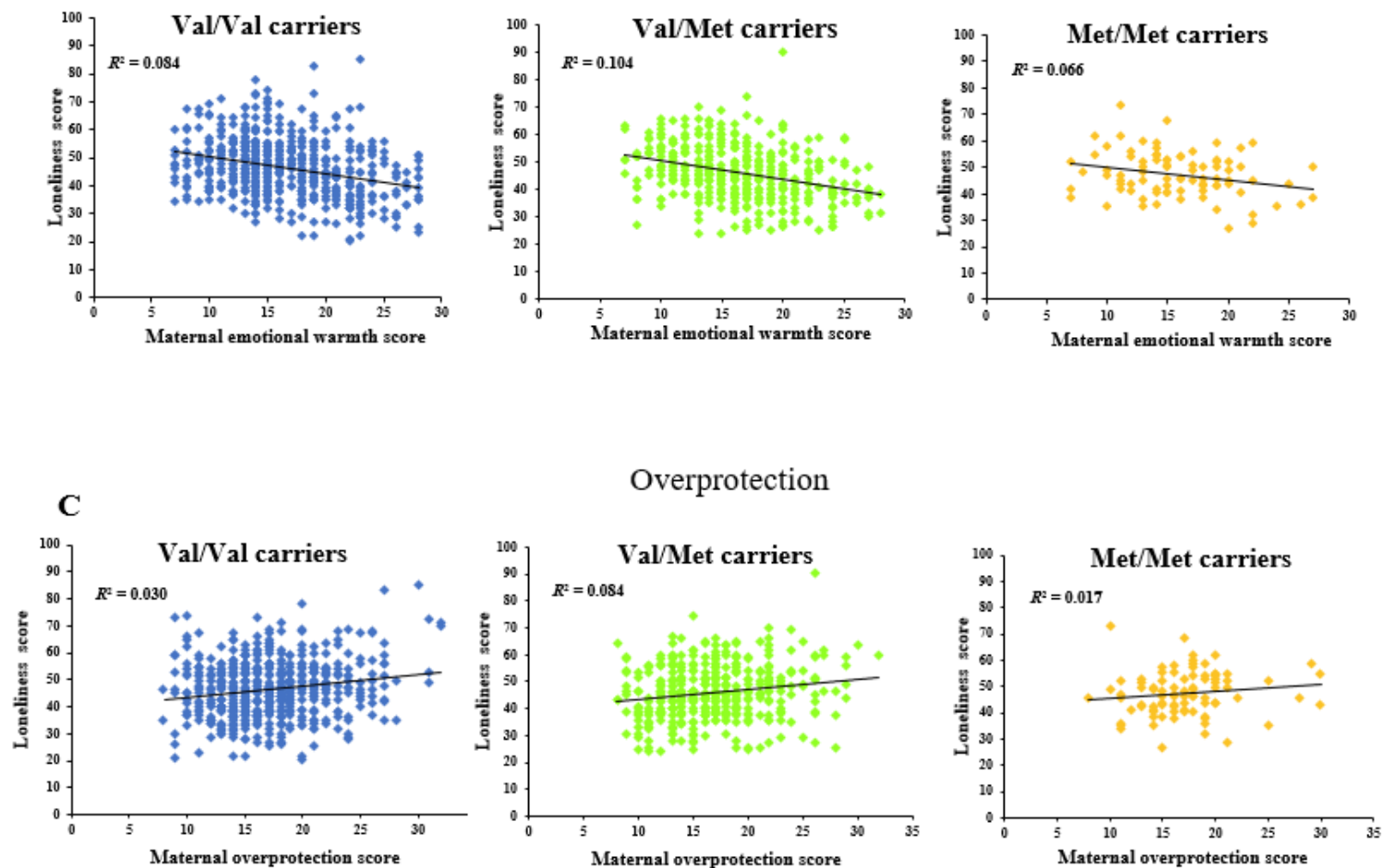

Fig. 2 The association between loneliness score and maternal support in the variants of COMT Val158Met.

Table 3. Results of the hierarchical regression analysis of mater support and loneliness. score in Study 1

\begin{tabular}{llllcccccc}
\hline & Variable & \multicolumn{1}{c}{ Sample } \\
\cline { 3 - 10 } & F change & $\mathrm{R}^{2}$ change & $\mathrm{B}$ & $S E$ & $t$ & $P$ & \multicolumn{2}{c}{ 95\% CI for B } \\
\hline $\begin{array}{l}\text { Reject } \\
\text { Step 1 }\end{array}$ & Gender & $37.63^{* * *}$ & 0.155 & -0.21 & 0.58 & -0.35 & 0.72 & -1.35 & 0.93
\end{tabular}




\begin{tabular}{|c|c|c|c|c|c|c|c|c|c|}
\hline & Age & & & -0.04 & 0.05 & -0.75 & 0.44 & -0.15 & 0.06 \\
\hline & Emotional warmth & & & $-0.51^{* * *}$ & 0.06 & -8.21 & 0.00 & -0.63 & -0.39 \\
\hline & Overprotection & & & $0.32^{* * * *}$ & 0.06 & 5.01 & 0.00 & 0.19 & 0.45 \\
\hline & SES & & & $-1.37^{* * *}$ & 0.20 & -6.87 & 0.00 & -1.76 & -0.97 \\
\hline \multirow[t]{2}{*}{ Step 2} & COMT & 13.28 & 0.02 & 0.17 & 0.44 & 0.39 & 0.69 & -0.69 & 1.04 \\
\hline & Reject & & & $0.55^{* * *}$ & 0.10 & 5.15 & 0.00 & 0.34 & 0.75 \\
\hline Step 3 & Reject $\times$ COMT & 6.17 & 0.005 & $-0.71^{*}$ & 0.28 & -2.48 & 0.01 & -1.27 & -0.15 \\
\hline \multicolumn{10}{|c|}{ Emotional warmth } \\
\hline \multirow[t]{5}{*}{ Step 1} & Gender & 30.78 & 0.13 & $-1.42^{*}$ & 0.59 & -2.39 & 0.01 & -2.59 & -0.25 \\
\hline & Age & & & 0.01 & 0.05 & -0.24 & 0.80 & -0.12 & 0.09 \\
\hline & Reject & & & $0.65^{* * *}$ & 0.10 & 6.05 & 0.00 & 0.44 & 0.86 \\
\hline & Overprotection & & & 0.11 & 0.07 & 1.47 & 0.14 & -0.03 & 0.26 \\
\hline & SES & & & $-1.61^{* * * *}$ & 0.20 & -8.22 & 0.00 & -2.01 & -1.23 \\
\hline \multirow[t]{2}{*}{ Step 2} & COMT & 28.54 & 0.17 & 0.17 & 0.44 & 0.39 & 0.69 & -0.69 & 1.04 \\
\hline & Emotional warmth & & & $-0.47^{* * *}$ & 0.06 & -7.52 & 0.00 & -0.59 & -0.34 \\
\hline Step 3 & $\begin{array}{l}\text { Emotional warmth } \times \\
\text { COMT }\end{array}$ & 0.136 & 0.17 & -0.10 & 0.29 & -0.37 & 0.71 & -0.67 & 0.46 \\
\hline \multicolumn{10}{|c|}{ Overprotection } \\
\hline \multirow[t]{5}{*}{ Step 1} & Gender & 43.20 & 0.17 & -0.86 & 0.58 & -1.48 & 0.14 & -2.00 & 0.28 \\
\hline & Age & & & -0.05 & 0.05 & -0.99 & 0.32 & -0.16 & 0.05 \\
\hline & Reject & & & $0.64^{* * * *}$ & 0.09 & 7.01 & 0.00 & 0.46 & 0.81 \\
\hline & Emotional warmth & & & $-0.47^{* * * *}$ & 0.06 & -7.50 & 0.00 & -0.58 & -0.34 \\
\hline & SES & & & $-1.30^{* * * *}$ & 0.120 & -6.60 & 0.00 & -1.69 & -0.91 \\
\hline \multirow[t]{2}{*}{ Step 2} & COMT & 1.51 & 0.17 & 0.17 & 0.44 & 0.39 & 0.69 & -0.69 & 1.04 \\
\hline & Overprotection & & & 0.12 & 0.07 & 1.69 & 0.09 & -0.02 & 0.27 \\
\hline Step 3 & $\begin{array}{l}\text { Overprotection } \times \\
\text { COMT }\end{array}$ & 0.18 & 0.17 & -0.12 & 0.29 & -0.43 & 0.66 & -0.69 & 0.44 \\
\hline
\end{tabular}

${ }^{*} p<.05,{ }^{* *} p<.01,{ }^{* * *} p<.001$.

COMT, catechol-O-methyltransferase; SES, Subjective socioeconomic status.

Taken together, the results indicate that there is a significant association between maternal reject and loneliness and this association is significantly moderated by COMT Val158Met. To test each hypothesis independently, we conducted a simple slopes analysis (Aiken \& West, 1991) and plotted the slopes at $1 S D$ above and below the mean (Figure 3). The simple slope test was significant in all three genotype groups (Val/Val: $\beta=0.74, t$ $=5.63, P<0.001 ; \mathrm{Val} / \mathrm{Met}: \beta=0.53, t=5.02, P<0.001 ; \mathrm{Met} / \mathrm{Met}: \beta=0.31, t=2.20, P$ $=0.02<.05 ;$ Figure 3), which means maternal reject scores has a significant predictive effect on ones' loneliness score under three conditions of COMT genotypes. These results also showed that when the number of Val allele increased, the unstandardized coefficient $(\beta)$ were increased. (Val/Val: $\beta=0.74>\mathrm{Val} / \mathrm{Met}: \beta=0.53>\operatorname{Met} /$ Met: $\beta=0.31$ ), which further revealed the moderation effect of COMT genotypes. 


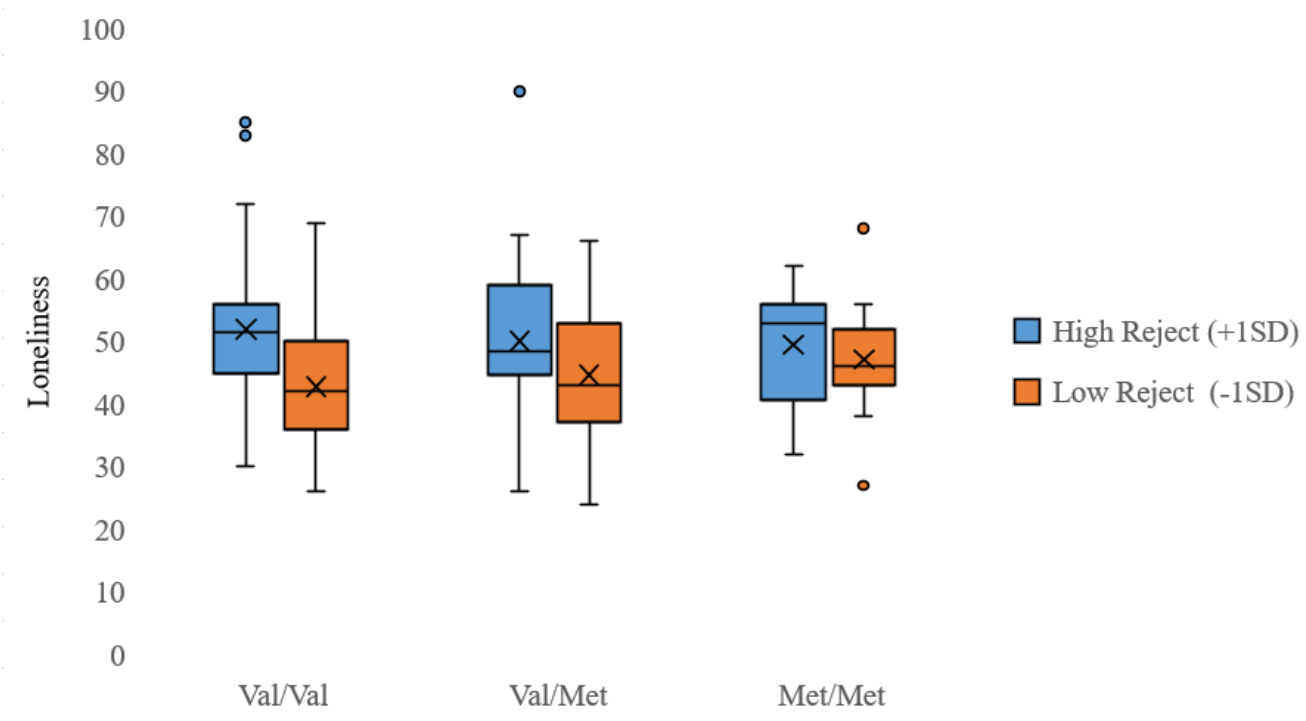

Fig. 3 Interactions between COMT Val158Met genotype and maternal rearing style.

\section{The COMT moderating effect across different age group}

The growing quantitative genetics literature shows that genetic influences on cognitive skills, like feeling loneliness, increase in magnitude over childhood, level out in adulthood, and they may decrease late in life, which inspires us to focus on the moderating effect over the entire life span. In particular, our hypothesis was that genes are a stronger predictor of loneliness than aging. To test the hypothesis, we divided the 1034 participants into three age groups: Group A (Age: 18 25), Group B (Age: 26 29) as well as Group C (Age: 30 50).

General Linear Model analysis with age, reject (high, low) and COMT revealed a main effect of maternal reject, $F(17,1034)=3.429, P<0.001$. The main effect of age $(F$ $(29,1034)=0.791, P=0.776)$ and $\operatorname{COMT}(F(2,1034)=1.758, P=0.173)$ were not significant. Analysis of three-way interaction showed that there wasn't a statistically interaction between COMT Val158 Met genotypes, maternal reject and age, $F(115,1034)$ $=1.037, P=0.388$. And the correlations between maternal reject and COMT were all significant in Val/Val genotype (Group A: $r=0.348, P<0.001$, Group B: $r=0.273, P=$ 0.001, Group C: $r=0.299, P<0.001)$. 
The COMT dependent mediation effect of loneliness between Maternal reject and Social Anxiety/Life Satisfaction

In this part, we explored the mediating role of loneliness and the moderating role of COMT Val158Met through maternal reject predicted social anxiety and life satisfaction.

Figure 4 shows the unstandardized regression coefficients for each path in the moderated mediation model. As seen in Fig. 4A, the test of moderated mediation revealed a significant interaction effect on loneliness $(B=-0.34, S E=0.13, t=-2.49, p=0.13)$. The index of moderated mediation was also significant which shows that the indirect effects in the model are significantly different at any two levels of COMT (index $=0.07, S E=0.03$, 95\% CI [0.01 0.13]).

The conditional indirect effect of maternal reject to social anxiety/life satisfaction through loneliness for indirect effects at three levels of COMT (Val/Val, Val/Met and Met/Met) was conducted by using PROCESS (Model 4). As seen in Fig. 4A, the conditional indirect effect of maternal reject to social anxiety through loneliness for indirect effect at three level of COMT (Val/Val, Val/Met and Met/Met) was only significant in $\mathrm{Val} / \mathrm{Val}$ level $(B=0.31, S E=0.07,95 \% \mathrm{CI}[0.180 .45])$. And the indirect effect was not significant at Val/ Met level ( $B=0.04, S E=0.04,95 \%$ CI [-0.43 0.15]) and Met/Met level $(B=0.15, S E=0.18,95 \%$ CI [-0.26 0.48]).

So, we further picked Val/Val level to continue our age-divided analysis. When the COMT variable was controlled to $\mathrm{Val} / \mathrm{Val}$ level, the mediation model was conducted at three age groups. The results have been shown in Table S1. As Table S1 demonstrates, the indirect effects were all significant.

As for life satisfaction, the conditional indirect effect was significant at Val/Val level $(B=-0.16, S E=0.03,95 \% \mathrm{CI}[-0.23-0.09])$. The indirect effect was not significant at Val/ Met level $(B=-0.04, S E=0.04,95 \%$ CI $[-0.130 .04])$ as well as at Met/Met level $(B=-$ $0.04, S E=0.07,95 \%$ CI [-0.19 0.11]) (Figure 4B).

When the COMT variable was controlled to Val/Val level, the mediation model was conducted at three age groups. The results have been shown in Table S1. As Table S1 demonstrates, the conditional direct effects were not significant in all age groups but the indirect effects were all significant. So, we can see that the pattern of the conditional 
indirect effects of COMT and age were just the same when the dependent variable was social anxiety.

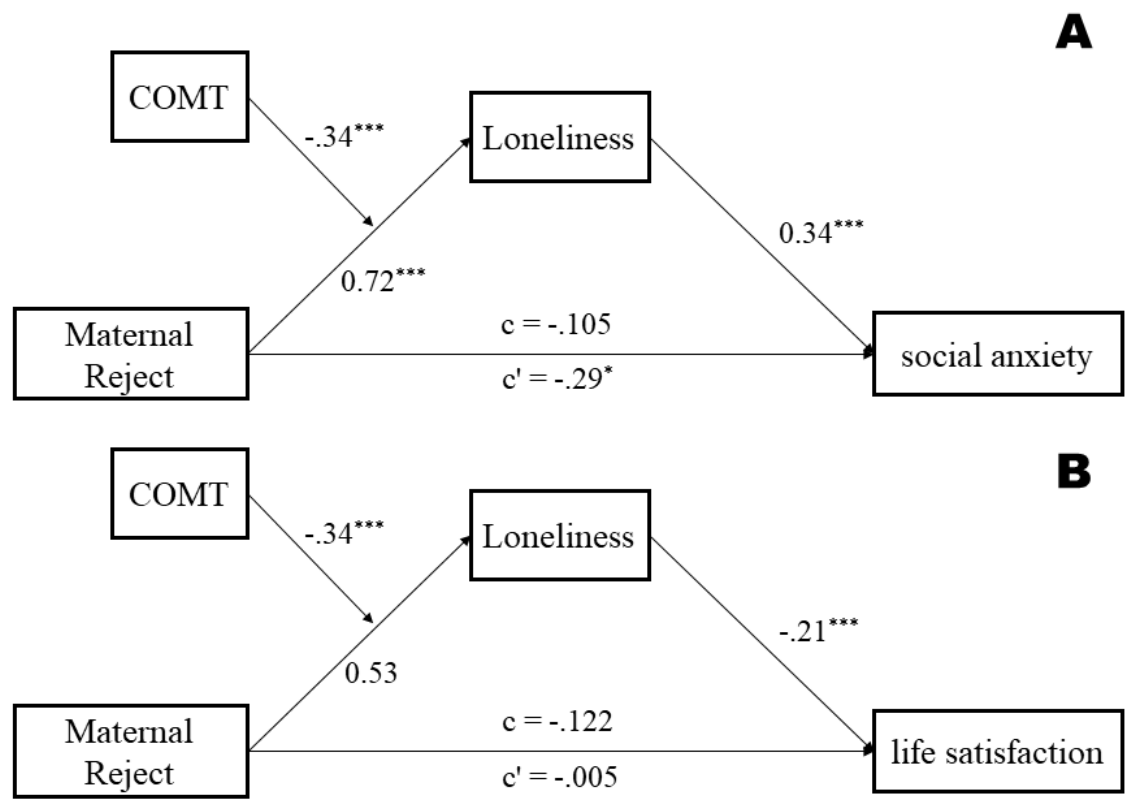

Fig. 4 Unstandardized regression coefficients for the moderated mediation model.

Note. ${ }^{* *} p<0.01$. The paths were run simultaneously in the analysis.

\section{Discussion}

In the present study, we aimed to conduct monogenic interaction $(\mathrm{G} \times \mathrm{E})$ analyses for adult loneliness development that involved genetic variation in the COMT Val158Met and maternal rearing style. We further investigated whether genetic and environmental interactions influence adult mental health through loneliness. The present results showed that COMT Val158Met polymorphism moderated the relationship between maternal rearing style and loneliness, but this moderating relationship existed only in maternal rejection, and we did not find statistical significance in emotional warmth and overprotective rearing style.

Adopting positive parenting rearing style, such as mother's emotional warmth, will make children feel less lonely in family, society and the whole (Zhou et al., 2013). Consistent with previous studies, the warm rearing style of mothers in this study did predict lower loneliness, but genes did not significantly modulate this prediction. One possible 
explanation is that the COMT gene is more sensitive to negative environments and less sensitive to positive ones like maternal warmth. Drury et al. (2010) found that the Met allele was only protective in the group of children who were raised in the adverse context of an institutional environment and the effect not apparent for those being raised in foster care families. This is similar to the findings of regarding the serotonin transporter gene where the short allele conferred vulnerability to depression, but only with significant negative life stress exposure (Caspi et al., 2003). Overprotective parents can make children less able and motivated to explore the outside world. However, children who grow up under overprotective parents can run back to their parents' arms in times of danger or difficulties and get enough support and love, so the feeling of loneliness may not be strong. This is one possible explanation for the main and moderating effects that we did not get from overprotection.

The results of the moderation model showed that the Val allele could significantly moderate maternal rejection rearing style and loneliness, while the Met/Met genotype had no significant moderating effect. Carriers of the Val allele showed higher levels of loneliness in the presence of a higher rejection of maternal parenting. However, the low rejection was associated with lower loneliness. For Met/Met genotypes, there was no significant difference in loneliness between high rejection and low rejection mothers. These results were consistent with Hosang's et al. (2017) studies, which find out the association between stressful life events and bipolar disorder is significant and higher for those in the Val dominant group compared to those with the Met/Met genotype. In other words, individuals with the Val allele were more susceptible to environmental factors, while Met/Met carriers were less susceptible to negative environmental factors. These results of this study are more consistent with the idea of $\mathrm{G} \times \mathrm{E}$ interaction in the different susceptibility models, that is, the genotype is not "bad genetic predisposition", but may simply be more sensitive and malleable to both favorable and unfavorable environments (Belsky \& Pluess, 2009; Ellis et al., 2011).

The main effects of maternal rejection and loneliness were significant, which aligns with Zhou's (2013) results showing that negative rearing style, such as mothers' rejection, will make children have more and more intense loneliness experiences. Meanwhile, we didn't find a significant correlation between genes and loneliness. One possible explanation 
for the non-significant direct effect of COMT Val158Met polymorphism in our sample on adults' loneliness is that the direct relationship between genes and complex psychological problems is usually small and therefore difficult to detect (Rutter, Moffitt, \& Caspi, 2006).

In the grouped control model, only individuals with the Val/Val genotype had significant moderating effects at all three ages. Ira et al. (2014) found that individuals exposed to low maternal care as a child (childhood adversity) reported more psychotic symptoms, and this relationship was strongest among the Val/Val COMT group. Carriers of the Val/Val gene might be more sensitive to negative environments and thus still had a significant moderating effect with age. However, there was no significant difference in the moderating effect between individuals with Val/Val genotype at different ages. In other words, the effects of genes are not overwhelmed in the rearing style's impact on loneliness by age's impacts. At the same time, the influence of maternal rejection on individual loneliness ran through the whole adult life. Curran (2019) studied participants aged 18-51, which reported higher levels of loneliness when their reports of familial social support were low. The results were significant across all age groups, suggesting that rearing style had a long-term, significant impact on adult loneliness.

When considering the mediation model, current findings revealed that COMT Val158Met moderated the association between maternal rearing style and loneliness in their relation to social anxiety and life satisfaction. Specifically, these completely mediated relations were evident among those with Val/Val genotype but absent in Met carriers. The current data were consistent with previous studies that carriers of the Val/Val allele were more sensitive to the negative environment (Hosang et al., 2017). Similarly, we tested mediating models with moderation in $\mathrm{Val} / \mathrm{Val}$ carriers in age groups. Results found out that in the presence of $\mathrm{Val} / \mathrm{Val}$ genotype, once loneliness was considered, the maternal rearing style no longer predicted social anxiety and life satisfaction in either age. These cross-age findings thus supported the argument that loneliness plays a central role in adult mental health, which specifically influences adult interpersonal and subjective well-being. Loneliness has been considered a major source of psychological stress (Friedler, Crapser, $\&$ McCullough, 2015), for instance, generalized anxiety, panic attacks and suicidality were strongly associated with loneliness. Loneliness poses significant health risks and in our study $\mathrm{G} \times \mathrm{E}$ interaction only influenced mental health through loneliness. Taken together, 
loneliness should be regarded and inquired about as a relevant health variable on its own (Heinrich, \& Gullone, 2006).

\section{Limitation and Expectation}

Although the present study indicated an association of the $\mathrm{G} \times \mathrm{E}$ interaction with loneliness and adult mental health, several limitations should be considered.

On the one hand, we only focus on maternal parenting as an environmental influence. Loneliness is a complex phenotype that may be the result of multiple environmental and biological factors. Stressful life events have been found to have a significant effect on loneliness (Segrin, 1999). Therefore, it is important to study the multiple environmental and genetic effects of loneliness in future large longitudinal sample studies. On the other hand, human problem behavior may be more susceptible to complex interactions, such as those between multiple genes and the environment, than the result of a single gene interacting with the environment. In future studies, we can further investigate the interaction between polygenes and the environment.

\section{References}

Aiken, L. S., West, S. G., \& Reno, R. R. (1991). Multiple regression: Testing and interpreting interactions. Sage.

Belsky, J., \& Pluess, M. (2009). Beyond diathesis stress: differential susceptibility to environmental influences. Psychological bulletin, 135(6), 885.

Cacioppo, J. T., \& Patrick, W. (2008). Loneliness: Human nature and the need for social connection. WW Norton \& Company.

Cacioppo, J. T., Cacioppo, S., \& Boomsma, D. I. (2014). Evolutionary mechanisms for loneliness. Cognition \& emotion, 28(1), 3-21.

Cacioppo, J. T., Hawkley, L. C., \& Thisted, R. A. (2010). Perceived social isolation makes me sad: 5year cross-lagged analyses of loneliness and depressive symptomatology in the Chicago Health, Aging, and Social Relations Study. Psychology and aging, 25(2), 453.

Caspi, A., Harrington, H., Moffitt, T. E., Milne, B. J., \& Poulton, R. (2006). Socially isolated children 20 years later: risk of cardiovascular disease. Archives of pediatrics \& adolescent medicine, 160(8), 805-811.

Caspi, A., Sugden, K., Moffitt, T.E., Taylor, A., Craig, I.W., Harrington, H., McClay, J., Mill, J., Martin, J., Braithwaite, A. \& Poulton, R. (2003) Influence of life stress on depression: moderation by a polymorphism in the 5-HTT gene. Science 301, 386-389.

Chao, R. C. L. (2012). Managing perceived stress among college students: The roles of social support and dysfunctional coping. Journal of College Counseling, 15(1), 5-21.

Cooney, T. M., \& Uhlenberg, P. (1992). Support from parents over the life course: The adult child's perspective. Social Forces, 71(1), 63-84.

Crockett, L. J., Iturbide, M. I., Torres Stone, R. A., McGinley, M., Raffaelli, M., \& Carlo, G. (2007). Acculturative stress, social support, and coping: Relations to psychological adjustment among Mexican American college students. Cultural Diversity and Ethnic Minority Psychology, 13(4), 347. 
Curran, T. (2019). Intergenerational transmissions of mother-child loneliness: A moderated mediation model of familial social support and conflict avoidance. Health communication, 34(10), 11661172.

Diener, Ed, Emmons, Robert A., Larsen, Randy J., \& Griffin, Sharon. . The satisfaction with life scale. $J$ Pers Assess, 49(1), 71-75.

DiTommaso, E., \& Spinner, B. (1993). The development and initial validation of the Social and Emotional Loneliness Scale for Adults (SELSA). Personality and Individual Differences, 14(1), 127-134.

Drury, S. S., Theall, K. P., Smyke, A. T., Keats, B. J., Egger, H. L., Nelson, C. A., ... \& Zeanah, C. H. (2010). Modification of depression by COMT val158met polymorphism in children exposed to early severe psychosocial deprivation. Child abuse \& neglect, 34(6), 387-395.

Dykstra, P. A., Van Tilburg, T. G., \& Gierveld, J. D. J. (2005). Changes in older adult loneliness: Results from a seven-year longitudinal study. Research on aging, 27(6), 725-747.

Ellis, B. J., Boyce, W. T., Belsky, J., Bakermans-Kranenburg, M. J., \& Van IJzendoorn, M. H. (2011). Differential susceptibility to the environment: An evolutionary-neurodevelopmental theory. Development and psychopathology, 23(1), 7-28.

Erzen, E., \& Çikrikci, Ö. (2018). The effect of loneliness on depression: A meta-analysis. International Journal of Social Psychiatry, 64(5), 427-435.

Faul, F., Erdfelder, E., Buchner, A., \& Lang, A. G. (2009). Statistical power analyses using G* Power 3.1: Tests for correlation and regression analyses. Behavior research methods, 41(4), 1149-1160.

Friedler, B., Crapser, J., \& McCullough, L. (2015). One is the deadliest number: the detrimental effects of social isolation on cerebrovascular diseases and cognition. Acta neuropathologica, 129(4), 493509.

Goodwin, R., Cook, O., \& Yung, Y. (2001). Loneliness and life satisfaction among three cultural groups. Personal Relationships, 8(2), 225-230.

Goossens, L., Van Roekel, E., Verhagen, M., Cacioppo, J. T., Cacioppo, S., Maes, M., \& Boomsma, D. I. (2015). The genetics of loneliness: Linking evolutionary theory to genome-wide genetics, epigenetics, and social science. Perspectives on Psychological Science, 10(2), 213-226.

Griffin, J. (2010). The lonely society. London, England: Mental Health Foundation.

Hawkley, L. C., Preacher, K. J., \& Cacioppo, J. T. (2007). Multilevel modeling of social interactions and mood in lonely and socially connected individuals: The MacArthur social neuroscience studies.

Hawthorne, G. (2008). Perceived social isolation in a community sample: its prevalence and correlates with aspects of peoples' lives. Social psychiatry and psychiatric epidemiology, 43(2), 140-150.

Hayes, A. F. (2015). An index and test of linear moderated mediation. Multivariate behavioral research, $50(1), 1-22$.

Hayes, A. F., \& Preacher, K. J. (2013). Conditional process modeling: Using structural equation modeling to examine contingent causal processes.

Heinrich, L. M., \& Gullone, E. (2006). The clinical significance of loneliness: A literature review. Clinical psychology review, 26(6), 695-718.

Hosang, G. M., Fisher, H. L., Cohen-Woods, S., McGuffin, P., \& Farmer, A. E. (2017). Stressful life events and catechol-O-methyl-transferase (COMT) gene in bipolar disorder. Depression and anxiety, 34(5), 419-426.

Ira, E., De Santi, K., Lasalvia, A., Bonetto, C., Zanatta, G., Cristofalo, D., ... \& Morandin, I. (2014). Positive symptoms in first-episode psychosis patients experiencing low maternal care and stressful life events: a pilot study to explore the role of the COMT gene. Stress, 17(5), 410-415.

Jiang, J., Lu, Z. R., Jiang, B. J., \& Xu, Y. (2010). Revision of the short-form Egna Minnen av Barndoms Uppfostran for Chinese. Psychological development and education, 1, 94-9.

Jones, W. H., Freemon, J. E., \& Goswick, R. A. (1981). The persistence of loneliness: Self and other determinants 1. Journal of personality, 49(1), 27-48.

Klein, M., Schmoeger, M., Kasper, S., \& Schosser, A. (2016). Meta-analysis of the COMT Val158Met polymorphism in major depressive disorder: the role of gender. The World Journal of Biological Psychiatry, 17(2), 147-158. 
Koch, M. J., \& McGrath, R. G. (1996). Improving labor productivity: Human resource management policies do matter. Strategic management journal, 17(5), 335-354.

Lan, W. H., Yang, A. C., Hwang, J. P., Hong, C. J., Liou, Y. J., Yeh, H. L., ... \& Tsai, S. J. (2012). Association of MTHFR C677T polymorphism with loneliness but not depression in cognitively normal elderly males. Neuroscience letters, 521(1), 88-91.

Leary, M. R., \& Kowalski, R. M. (1993). The interaction anxiousness scale: Construct and criterionrelated validity. Journal of personality assessment, 61(1), 136-146.

Lee, C., Dickson, D. A., Conley, C. S., \& Holmbeck, G. N. (2014). A closer look at self-esteem, perceived social support, and coping strategy: A prospective study of depressive symptomatology across the transition to college. Journal of Social and Clinical Psychology, 33(6), 560-585.

Lim, M. H., Rodebaugh, T. L., Zyphur, M. J., \& Gleeson, J. F. (2016). Loneliness over time: The crucial role of social anxiety. Journal of abnormal psychology, 125(5), 620.

Lucht, M. J., Barnow, S., Sonnenfeld, C., Rosenberger, A., Grabe, H. J., Schroeder, W., ... \& Rosskopf, D. (2009). Associations between the oxytocin receptor gene (OXTR) and affect, loneliness and intelligence in normal subjects. Progress in Neuro-Psychopharmacology and Biological Psychiatry, 33(5), 860-866.

Luo, Y., Hawkley, L. C., Waite, L. J., \& Cacioppo, J. T. (2012). Loneliness, health, and mortality in old age: A national longitudinal study. Social science \& medicine, 74(6), 907-914.

Mahon, N. E., Yarcheski, A., Yarcheski, T. J., Cannella, B. L., \& Hanks, M. M. (2006). A meta-analytic study of predictors for loneliness during adolescence. Nursing research, 55(5), 308-315.

Rutter, M., Moffitt, T. E., \& Caspi, A. (2006). Gene-environment interplay and psychopathology: Multiple varieties but real effects. Journal of child Psychology and Psychiatry, 47(3-4), 226-261.

Segrin, C. (1999). Social skills, stressful life events, and the development of psychosocial problems. Journal of Social and Clinical Psychology, 18(1), 14-34.

Tsai, S. J., Yeh, H. L., Hong, C. J., Liou, Y. J., Yang, A. C., Liu, M. E., \& Hwang, J. P. (2012). Association of CHRNA4 polymorphism with depression and loneliness in elderly males. Genes, Brain and Behavior, 11(2), 230-234.

van Roekel, E., Scholte, R. H., Verhagen, M., Goossens, L., \& Engels, R. C. (2010). Loneliness in adolescence: Genex environment interactions involving the serotonin transporter gene. Journal of Child Psychology and Psychiatry, 51(7), 747-754.

Xu, J., Ni, S., Ran, M., \& Zhang, C. (2017). The relationship between parenting styles and adolescents' social anxiety in migrant families: A study in Guangdong, China. Frontiers in psychology, 8, 626.

Yavuzer, Y., Albayrak, G., \& Kilıçarslan, S. (2019). Relationships amongst aggression, self-theory, loneliness, and depression in emerging adults. Psychological reports, 122(4), 1235-1258.

Yeung, W. J., Sandberg, J. F., Davis-Kean, P. E., \& Hofferth, S. L. (2001). Children's time with fathers in intact families. Journal of Marriage and Family, 63(1), 136-154.

Zhou, X., Zhu, H., Zhang, B., \& Cai, T. (2013). Perceived social support as moderator of perfectionism, depression, and anxiety in college students. Social Behavior and Personality: an international journal, 41(7), 1141-1152. 